\title{
Hyaluronic acid microinjection effective for facial aging treatment in lower periorbital area
}

New study demonstrates appropriate application technique for derma filler Restylane Vital Skinbooster to boost the aging under-eye region

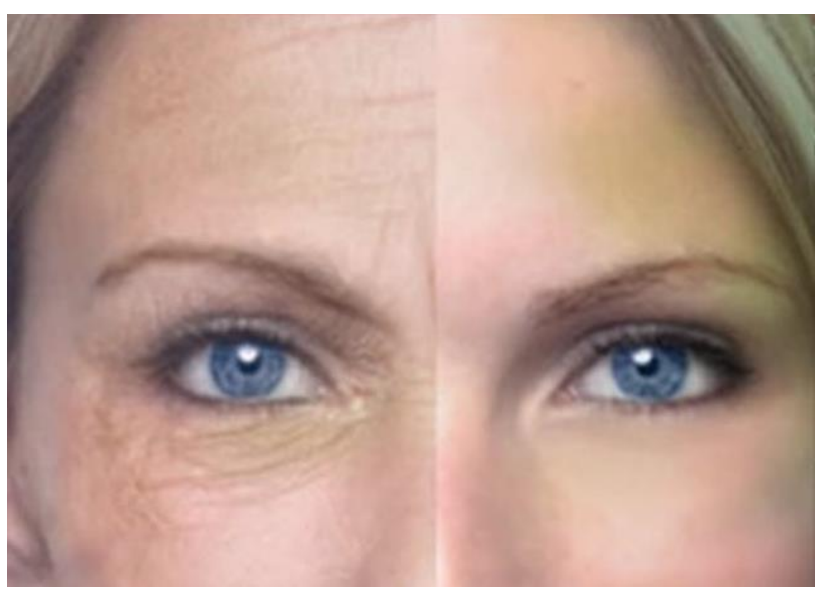

$\mathrm{T}$ he use of injectable dermal filler hyaluronic acid has proved efficient in the treatment of facial aging in the lower periorbital area (LPOA), according to a team of Brazilian researchers in a study published by the Journal of Surgical Dermatology.

"Our data suggests that the use of Restylane Vital Skinbooster along the LPOA presents a low risk of complications, is easy to perform, and provides a high satisfaction degree," reported Dr. Denis Souto Valente from the Dermatologic Surgery Department of the Mãe de Deus Health System, Porto Alegre, Brazil, and his fellow dermatology researchers.
The lower periorbital - the underside of the region surrounding the eye socket-is a vital area for facial rejuvenation, and changes in the periorbital during the aging process typically involve loss of volume, sagging skin, dark circles and discoloration, which often resulted in a tired, hooded appearance. Hence it is unsurprising that its aesthetic reconstructions are often so popularly requested. For instance, facial anti-aging skin care products were the second highest grossing skin care category in the United States in 2014, with consumers spending an excess of USD $\$ 1.15$ billion dollars.

Non-surgical therapies chiefly procedures using hyaluronic acid (HA) have already been studied, and previous research have established it to be a safe option as an injectable dermal filler, according to Valente and his research team. "HA also rarely induces significant side effects," they described, adding that HA's complications mostly disappear when it disintegrates or is catalyzed by the natural enzyme hyaluronidase.

Hyaluronic acid, which despite its name is actually a natural sugar instead of an acid, is a compound found in the skin and connective tissues, with the function of maintaining cell volume and pliability, along with serving as a cushion and lubricant for joints, nerves, hair, skin and eyes. As dehydration is one of the main causes behind skin aging, HA with its "humectant" properties - molecules that attracts and retains water-would repair the skin's moisture barrier by filling up the spaces between the collagen and elastin in the skin, consequently helping the skin stay supple while preventing wrinkles from forming.

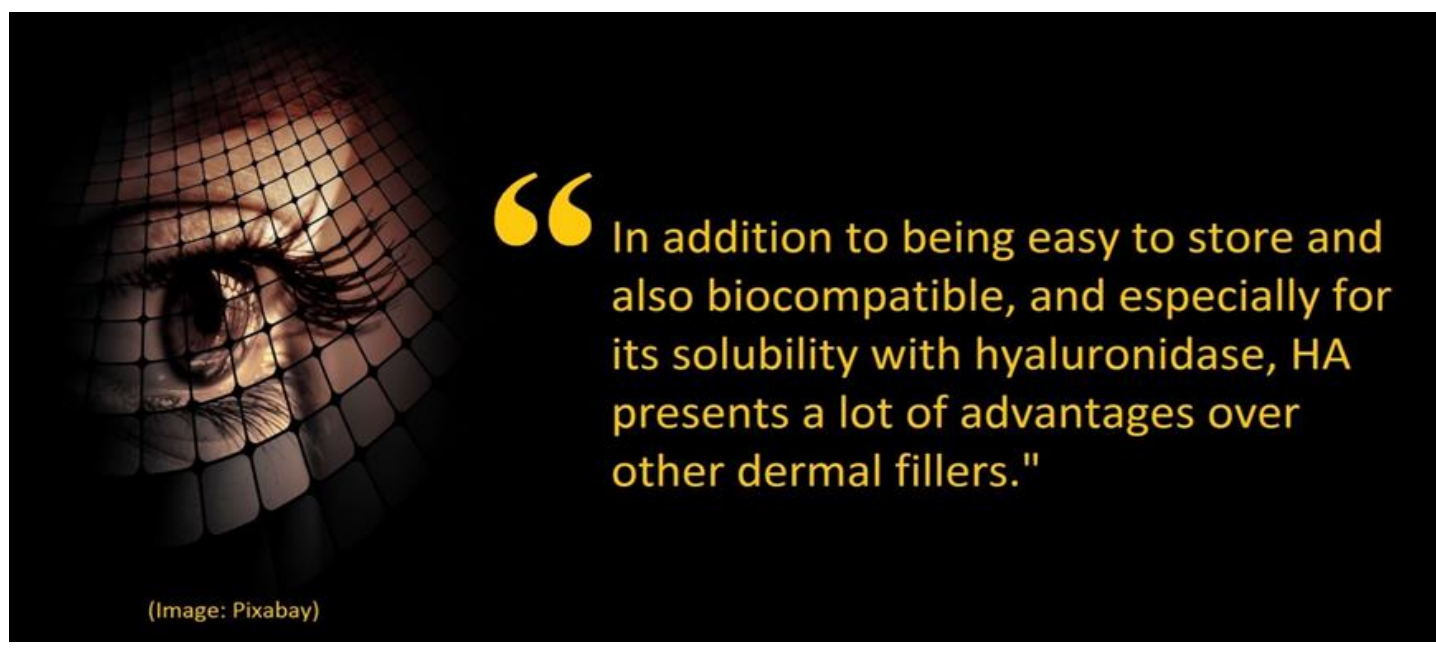




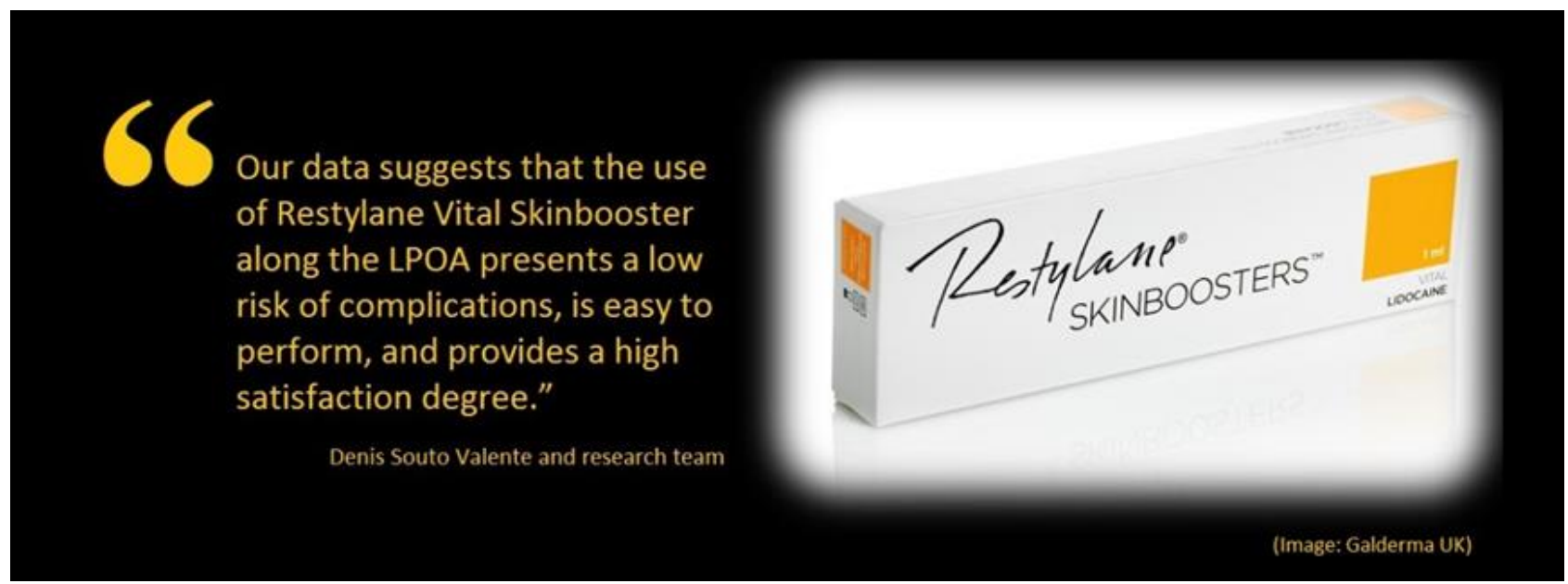

Furthermore, HA's homogeneous texture, low allergenic potential and simplicity in application "provides lower complication rates and predictable results when applied to the skin layer," according to the authors. "In addition to being easy to store and also biocompatible, and especially for its solubility with hyaluronidase, HA presents a lot of advantages over other dermal fillers," they noted.

Restylane, a transparent filler developed by Swedish medical company Q-Med in 1996, is a patented form of FDA-approved NASHA (or Non-Animal Stabilized Hyaluronic Acid) containing the so-called "stabilized HA"- the soft and gel-like water-absorbing substance similar to human body's own hyaluronic acid, yet "have a dramatically longer shelf life allowing for much longer hydration in the skin," according to its manufacturer.

The product Restylane Vital Skinbooster, meanwhile, "was developed to meliorate skin structure, restore its hydrobalance, and improve elasticity," according to Valente and his research team. The product is essentially a new concept of using Restylane: rather than injecting the HA into particular parts of the face to fill up sagging areas affected by aging, a series of micro amounts of Restylane are injected into larger areas of the skin, hence adding an overall boost to the face.

However, despite the well-known use of HA in skin therapy, not much information is available of its effectiveness in treating particularly the lower periorbital area. "The LPOA is a challenging area for treatments" owing to LPOA's tenuous and vascularized skin, according to the authors. "Due to the unique anatomical features of LPOA, we did not find any consensus in literature regarding the most suitable technique for HA application along this area," the researchers said.

Hence, their study sought to examine the effectiveness and safety of the technique for LPOA filling of a single HA application at the superficial layer. Using assessment by autonomous observers examining before-and-after photos of the studied patients, along with patients' own self-assessment using a visual analog scale, the study found that the independent physicians' evaluation presented a superb 78.4 mean improvement rate on a scale of 0 (no enhancement) to 100 (maximum enhancement).

Meanwhile, patient's own evaluations post-treatment resulted in an overwhelming majority of $90 \%$ reporting moderate-to-great improvement, with $0 \%$ reporting no improvement. Aside from the glowing satisfaction from patients and the positive assessment results by the physicians, HA injection technique described in the paper "allows a constant visualization of the needle in order to assert that the HA is being injected along the correct layer," said the authors, adding that Restylane Vital Skinbooster infiltration at the lower periorbital area also proved to be a safe treatment.

The research team includes Denis Souto Valente, Rafaela Koehler Zanella, Ângelo Syrillo Preto Neto, Sibelie Souto Valente and Felipe Laranjeira. Their original research article is published in this issue of JSD (page 168-174) and can be downloaded at: http://www.jsurgdermatol.com/ 